\title{
EXTINCTION TIMES IN MULTITYPE MARKOV BRANCHING PROCESSES
}

\author{
DOMINIK HEINZMANN, ${ }^{*}$ University of Zurich
}

\begin{abstract}
In this paper, a distributional approximation to the time to extinction in a subcritical continuous-time Markov branching process is derived. A limit theorem for this distribution is established and the error in the approximation is quantified. The accuracy of the approximation is illustrated in an epidemiological example. Since Markov branching processes serve as approximations to nonlinear epidemic processes in the initial and final stages, our results can also be used to describe the time to extinction for such processes.
\end{abstract}

Keywords: Multitype branching process; extinction time; convergence rate

2000 Mathematics Subject Classification: Primary 60J80

Secondary 92D30

\section{Introduction}

This paper is concerned with approximating the time to extinction in a subcritical multitype Markov branching process, starting with many individuals. The argument is based on the classical exponential approximation to the extinction probabilities [1], [11]-[13], [17]. These approximations are then combined with the branching property to derive a Gumbel approximation. The bound on the error in the total variation distance is inversely proportional to a positive power of a weighted sum of the number of individuals of the different types. The power depends on the means and higher moments of the offspring distribution.

In infectious disease modeling, the initial and final stages of epidemic processes can often be approximated by suitable branching processes; see [18]. More recently, in [2]-[5], different constructions have been used to quantify the path accuracy of such approximations. These results can be combined with ours to derive corresponding statements about the extinction time in epidemic processes.

\section{Equations for extinction probabilities}

The notation is chosen with [1, p. 200], [11, p. 113], and [17, p. 77] as basic references. For $k<\infty$, set $\boldsymbol{Z}(t)=\left(Z_{1}(t), \ldots, Z_{k}(t)\right)$, where $Z_{i}(t)$ is the number of individuals of type $i$ at time $t$. A type $i$ individual has exponential lifetime with parameter $a_{i}$ and rises at death $j_{i}$ type $i$ individuals, $1 \leq i \leq k$, with probability $p_{i}^{j}$, where $\boldsymbol{j}=\left(j_{1}, \ldots, j_{k}\right) \in \mathbb{Z}_{+}^{k}$, independent of everything that has happened up to this time. Assume that

$$
R_{i l}:=\sum_{j \in \mathbb{N}^{k}} p_{i}^{j} j_{l}<\infty \quad \text { for } i \geq 1 \text { and } l \leq k .
$$

Received 15 August 2008; revision received 4 November 2008.

* Postal address: Institute of Mathematics, University of Zurich, Winterthurerstrasse 190, 8057 Zurich, Switzerland.

Email address: dominik.heinzmann@math.uzh.ch 
Let $\|\cdot\|$ be the supremum norm, and let $\mathrm{P}_{\boldsymbol{I}}$ be a conditional distribution of the process at time $t$ given $\boldsymbol{Z}(0)=\boldsymbol{I}$ for $\boldsymbol{I}=\left(I_{1}, \ldots, I_{k}\right) \in \mathbb{Z}_{+}^{k}$. In particular, let $\mathrm{P}_{i}$ correspond to the case when $Z_{i}(0)=1$ and $Z_{m}(0)=0, m \neq i$. Let $T$ be the extinction time of the process, and define the survival probability of the process when starting with a single type $i$ individual as $q_{i}(t):=1-\mathrm{P}_{i}(T \leq t)=1-\mathrm{P}_{i}(\boldsymbol{Z}(t)=\mathbf{0})$.

Then Equation (15.2) of [11, p. 114] implies that

$$
\frac{1}{a_{i}} \frac{\mathrm{d}}{\mathrm{d} t}\left(1-q_{i}(t)\right)=q_{i}(t)-\sum_{j \in \mathbb{N}^{k}} p_{i}^{j}\left(1-\prod_{l=1}^{k}\left(1-q_{l}(t)\right)^{j_{l}}\right) \quad \text { for } 1 \leq i \leq k .
$$

Now the relation $(1-x)^{l} \geq 1-x l$ for $0 \leq x \leq 1$, together with the Bonferroni inequalities [7, p. 27], implies that

$$
\sum_{\boldsymbol{j} \in \mathbb{N}^{k}} p_{i}^{j}\left(1-\prod_{l=1}^{k}\left(1-q_{l}(t)\right)^{j_{l}}\right) \leq \sum_{\boldsymbol{j} \in \mathbb{N}^{k}} p_{i}^{j}\left(\boldsymbol{j}^{\top} \boldsymbol{q}(t)\right)
$$

and

$$
\sum_{\boldsymbol{j} \in \mathbb{N}^{k}} p_{i}^{j}\left(1-\prod_{l=1}^{k}\left(1-q_{l}(t)\right)^{j_{l}}\right) \geq \sum_{\boldsymbol{j} \in \mathbb{N}^{k}} p_{i}^{j} \max \left\{\boldsymbol{j}^{\top} \boldsymbol{q}(t)-F^{j}(\boldsymbol{q}(t)), 0\right\},
$$

where $\boldsymbol{q}(t)=\left(q_{1}(t), \ldots, q_{k}(t)\right)$ and

$$
F^{j}(\boldsymbol{q}(t)):=\frac{1}{2} \sum_{\substack{l, l^{\prime}=1 \\ l \neq l^{\prime}}}^{k} j_{l} j_{l^{\prime}} q_{l}(t) q_{l^{\prime}}(t)+\frac{1}{2} \sum_{l=1}^{k} j_{l}\left(j_{l}-1\right) q_{l}^{2}(t) \leq \frac{1}{2}\left(\boldsymbol{j}^{\top} \boldsymbol{q}(t)\right)^{2} .
$$

Using (2.3) and (2.4) in (2.2) and recalling (2.1) gives

$$
\frac{1}{a_{i}} \frac{\mathrm{d} q_{i}(t)}{\mathrm{d} t} \leq\{(\boldsymbol{R}-\boldsymbol{I}) \boldsymbol{q}(t)\}_{i}
$$

and

$$
\frac{1}{a_{i}} \frac{\mathrm{d} q_{i}(t)}{\mathrm{d} t}=\{(\boldsymbol{R}-\boldsymbol{I}) \boldsymbol{q}(t)\}_{i}-v_{i}(t)
$$

where $v_{i}(t)$ summarizes all the nonlinear terms in $\boldsymbol{q}(t)$ (see Section 5 for an example) and satisfies

$$
\begin{aligned}
0 & \leq v_{i}(t) \\
& =\sum_{\boldsymbol{j} \in \mathbb{N}^{k}} p_{i}^{j}\left(\sum_{l=1}^{k} j_{l} q_{l}(t)-1+\prod_{l=1}^{k}\left(1-q_{l}(t)\right)^{j_{l}}\right) \\
& \leq \sum_{\boldsymbol{j} \in \mathbb{N}^{k}} p_{i}^{j} \min \left\{F^{j}(\boldsymbol{q}(t)), \boldsymbol{j}^{\top} \boldsymbol{q}(t)\right\} .
\end{aligned}
$$

Since (2.2) is nonlinear in $\boldsymbol{q}(t)$, it cannot in general be solved analytically. However, we will see, using Theorem 3.1, below, that the behavior of the solution $\boldsymbol{q}(t)$ can be approximated by that of

$$
\frac{\mathrm{d} \boldsymbol{q}(t)}{\mathrm{d} t}=\{\boldsymbol{A}(\boldsymbol{R}-\boldsymbol{I})\} \boldsymbol{q}(t)=: \boldsymbol{B} \boldsymbol{q}(t)
$$


so long as $\|\boldsymbol{q}(t)\|$ is small and $\boldsymbol{A}:=\operatorname{diag}\left\{a_{1}, \ldots, a_{k}\right\}$. The matrix $\boldsymbol{B}=\boldsymbol{A}(\boldsymbol{R}-\boldsymbol{I})$ has nonnegative elements off the diagonal, and is thus a Metzler-Leontief matrix [16, p. 40]. If $\boldsymbol{B}$ is irreducible [16, p. 15], the process $\boldsymbol{Z}(t)$ is irreducible [17, p. 99] and the following PerronFröbenius result [16, Theorem 2.5] applies.

Theorem 2.1. Assume that $\boldsymbol{B}$ is a $k \times k$ irreducible matrix with nonnegative off-diagonal elements. Then there exists an eigenvalue $\omega$ such that

(i) $\omega$ is real;

(ii) there exists a unique (up to a constant factor) strictly positive left eigenvector $f_{1}$ and a unique strictly positive right eigenvector $\boldsymbol{b}_{1}$ associated with $\omega$;

(iii) $\omega>\operatorname{Re}\left(\omega_{i}\right)$ for any eigenvalue $\omega_{i} \neq \omega$ of $\boldsymbol{B}$;

(iv) $\omega$ is a simple root of the characteristic equation of $\boldsymbol{B}$.

In what follows, it is assumed that the process is subcritical, i.e. $\omega<0$. Define $r:=-\omega$. The left eigenvector $f_{1}$ can be used to derive an upper bound for $\boldsymbol{q}(t)(t>0)$.

Lemma 2.1. Assume that $\boldsymbol{f}_{1}^{\top}=\left(f_{11}, \ldots, f_{1 k}\right)$ is such that $\left\|\boldsymbol{f}_{1}\right\|=1$. Then

$$
q_{i}(t) \leq \mathrm{e}^{-r t}\left(\frac{f_{1}^{\top} \mathbf{1}}{f_{1 i}}\right) \quad \text { for } 1 \leq i \leq k,
$$

where 1 denotes a column vector of $1 s$.

Proof. Theorem 2.1 implies that $f_{1}$ has only positive entries and, hence, inequality (2.5) implies that

$$
\frac{\mathrm{d}}{\mathrm{d} t}\left\{\boldsymbol{f}_{1}^{\top} \boldsymbol{q}(t)\right\} \leq \boldsymbol{f}_{1}^{\top} \boldsymbol{B} \boldsymbol{q}(t)=\omega \boldsymbol{f}_{1}^{\top} \boldsymbol{q}(t)=-r \boldsymbol{f}_{1}^{\top} \boldsymbol{q}(t) .
$$

Using Grönwall's lemma [10] yields

$$
\boldsymbol{f}_{1}^{\top} \boldsymbol{q}(t) \leq \mathrm{e}^{-r t} \boldsymbol{f}_{1}^{\top} \boldsymbol{q}(0)=\mathrm{e}^{-r t} \boldsymbol{f}_{1}^{\top} \mathbf{1}
$$

The result follows immediately, since $f_{1}$ and $\boldsymbol{q}(t)$ are both positive vectors.

The following useful lemma is proved by a standard argument.

Lemma 2.2. Let $X$ be a nonnegative random variable with $\mathrm{E}(X)<\infty$, and let $d>1$. Then, for $\delta \rightarrow 0, \mathrm{E}\left(X \delta \wedge(X \delta)^{d}\right)=o(\delta)$. If, in addition, $\mathrm{E}\left(X^{\psi}\right)<\infty$ for some $1 \leq \psi \leq d$ then $\mathrm{E}\left(X \delta \wedge(X \delta)^{d}\right) \leq 2 \mathrm{E}(X \delta)^{\psi}=O\left(\delta^{\psi}\right)$.

Let $J_{i}$ denote a random variable with $\mathrm{P}\left(J_{i}=j\right)=p_{i}^{j}$.

Theorem 2.2. If $\mathrm{E}\left(\left\|J_{i}\right\|\right)<\infty$ for $1 \leq i \leq k$ then $v_{i}(t)=o(\|\boldsymbol{q}(t)\|)$ as $t \rightarrow \infty$.

Proof. From (2.7), it follows that

$$
0 \leq v_{i}(t) \leq \sum_{j \in \mathbb{N}^{k}} p_{i}^{j}\left(\frac{1}{2}\left(\boldsymbol{j}^{\top} \boldsymbol{q}(t)\right)^{2} \wedge\left(\boldsymbol{j}^{\top} \boldsymbol{q}(t)\right)\right)=\mathrm{E}\left(\frac{1}{2}\left(J^{\top} \boldsymbol{q}(t)\right)^{2} \wedge\left(\boldsymbol{J}^{\top} \boldsymbol{q}(t)\right)\right),
$$

where $\boldsymbol{J}^{\top}=\left(J_{1}, \ldots, J_{k}\right)$. Since $\|\boldsymbol{q}(t)\| \leq \sum_{i=1}^{k}\left|q_{i}(t)\right|$, Lemma 2.1 indicates that $\|\boldsymbol{q}(t)\| \rightarrow 0$ as $t \rightarrow \infty$, and, thus, Lemma 2.2 can be applied.

The following corollary gives a more specific asymptotic upper bound on $v_{i}(t)$, if the offspring distributions have a finite moment higher than the first. 
Corollary 2.1. Suppose that $\mathrm{E}\left(\left\|J_{i}\right\|^{1+\alpha}\right)<\infty$ for some $0<\alpha \leq 1$ and all $1 \leq i \leq k$. Then there exist constants $c_{i}^{*}<\infty$ such that $0 \leq v_{i}(t) \leq c_{i}^{*}\|\boldsymbol{q}(t)\|^{1+\alpha}, 1 \leq i \leq k$.

Proof. The proof follows immediately from (2.8) and Lemma 2.2.

\section{Exponential limit behavior}

The following result is the basis for approximating the survival time of the process, bounding the error in the exponential approximation to the extinction probabilities.

Theorem 3.1. Assume that $\mathrm{E}\left(\left\|J_{i}\right\|^{1+\alpha}\right)<\infty$ for some $0<\alpha \leq 1$ and all $1 \leq i \leq k$. If $\boldsymbol{B}$ is irreducible with largest eigenvalue $-r<0$, the probability of survival $q_{i}(t)$ when starting with a single individual of type $i$ satisfies

$$
q_{i}(t)=c_{i} \mathrm{e}^{-r t}\left(1+o\left(\mathrm{e}^{-\gamma t}\right)\right)
$$

where $0<\gamma<r$ is given below and $c_{i} / c_{l}=b_{1 i} / b_{1 l}$, where $\boldsymbol{b}_{1}$ is the right eigenvector of $\boldsymbol{B}$ corresponding to the eigenvalue $-r$.

Proof. Let $\boldsymbol{v}(t):=\left(v_{1}(t), \ldots, v_{k}(t)\right)$, and define $\boldsymbol{u}(t):=\mathrm{e}^{r t} \boldsymbol{q}(t)$. It follows from (2.6) that

$$
\frac{\mathrm{d}}{\mathrm{d} t} \boldsymbol{u}(t)=\boldsymbol{C} \boldsymbol{u}(t)-\mathrm{e}^{r t} \boldsymbol{A} \boldsymbol{v}(t),
$$

where the largest eigenvalue of $\boldsymbol{C}:=(\boldsymbol{B}+r \boldsymbol{I})$ is 0 . Let 0 and $\left\{\omega_{j} ; 2 \leq j \leq k^{*}\right\}$ denote the eigenvalues corresponding to the $k^{*} \leq k$ Jordan blocks of $\boldsymbol{C}$, and denote by $k_{j}, 2 \leq j \leq k^{*}$, their dimensions. The left eigenvector of $\boldsymbol{C}$ corresponding to the eigenvalue 0 is $\boldsymbol{f}_{1}^{\top}$; for $1 \leq m \leq k_{j}$ and $2 \leq j \leq k^{*}$, let $\boldsymbol{f}_{i, m}^{\top}$ denote the corresponding Jordan basis vectors, with $\left\|\boldsymbol{f}_{j, m}\right\|=1$; set $-\beta_{j}=\operatorname{Re}\left(\omega_{j}\right)$, so that, for $2 \leq m \leq k_{j}$ and $2 \leq j \leq k^{*}, \boldsymbol{f}_{j, 1}^{\top} \boldsymbol{C}=\omega_{j} \boldsymbol{f}_{j, 1}^{\top}$ and $\boldsymbol{f}_{j, m}^{\top} \boldsymbol{C}=\omega_{j} \boldsymbol{f}_{j, m}^{\top}+\boldsymbol{f}_{j, m-1}^{\top}$.

Define $\boldsymbol{w}(t):=\boldsymbol{f}_{1}^{\top} \boldsymbol{A} \boldsymbol{v}(t) /\left(\boldsymbol{f}_{1}^{\top} \boldsymbol{q}(t)\right)$. From Lemma 2.1 and Corollary 2.1, it is immediate that $\|\boldsymbol{w}(t)\|=O\left(\mathrm{e}^{-r \alpha t}\right)$ and, hence, that $\int_{s}^{\infty}\|\boldsymbol{w}(t)\| \mathrm{d} t<\infty$.

Now, from (3.1),

$$
\frac{\mathrm{d}}{\mathrm{d} t} \log \left(\boldsymbol{f}_{1}^{\top} \boldsymbol{u}(t)\right)=-\boldsymbol{w}(t)
$$

and, hence,

$$
\log \left(\boldsymbol{f}_{1}^{\top} \boldsymbol{q}(t)\right)+r t=\log \left(\boldsymbol{f}_{1}^{\top} \boldsymbol{q}(s)\right)+r s-\int_{s}^{t} \boldsymbol{w}(z) \mathrm{d} z .
$$

By the Cauchy criterion and the integrability of $\|\boldsymbol{w}(z)\|$, it follows that

$$
\lim _{t \rightarrow \infty}\left\{\log \left(\boldsymbol{f}_{1}^{\top} \boldsymbol{q}(t)\right)+r t\right\}=: \log h^{*}
$$

exists and is finite, and, thus, using $\|\boldsymbol{w}(t)\|=O\left(\mathrm{e}^{-r \alpha t}\right)$,

$$
\boldsymbol{f}_{1}^{\top} \boldsymbol{q}(t)=h^{*} \mathrm{e}^{-r t}\left(1+O\left(\mathrm{e}^{-r \alpha t}\right)\right)
$$

with $h^{*}>0$.

For the remaining part of the argument, we refer to the theory of perturbed linear systems. Rewrite (3.1) as

$$
\frac{\mathrm{d}}{\mathrm{d} t} \boldsymbol{u}(t)=[\boldsymbol{C}+\boldsymbol{D}(t)] \boldsymbol{u}(t),
$$


where

$$
\boldsymbol{D}(t)=-\frac{\boldsymbol{A} \boldsymbol{v}(t) \boldsymbol{q}(t)^{\top}}{\|\boldsymbol{q}(t)\|^{2}}
$$

so that $\|\boldsymbol{D}(t)\| \leq K^{*} \mathrm{e}^{-r \alpha t}$ with $K^{*}<\infty$. System (3.2) is a special case of the system in Theorem 2 of [14], from which it follows that, for any $\gamma<\min \left\{r \alpha, \beta_{[2]}\right\}$, where $-\beta_{[2]}$ is the second largest real part of any eigenvalue of $\boldsymbol{C}$, we have $\left|\boldsymbol{f}_{j, m}^{\top} \boldsymbol{u}(t)\right|=o\left(\mathrm{e}^{-\gamma t}\right), 1 \leq m \leq k_{j}$ and $2 \leq j \leq k^{*}$.

Now the set of vectors $\left\{f_{1}^{\top}, f_{j, m}^{\top} ; 1 \leq m \leq k_{j}, 2 \leq j \leq k^{*}\right\}$ constitutes a basis of $\mathbb{R}^{d}$. Let $\boldsymbol{x} \in \mathbb{R}^{d}$ have coefficients $\left(x_{1}, x_{j, m} ; 1 \leq m \leq k_{j}, 2 \leq j \leq k^{*}\right)$ with respect to this basis. Then

$$
\boldsymbol{x}^{\top}\left(\mathrm{e}^{r t} \boldsymbol{q}(t)\right)=\left(x_{1} \boldsymbol{f}_{1}^{\top}+\sum_{j=2}^{k^{*}} \sum_{n=1}^{k_{j}} x_{j, n} \boldsymbol{f}_{j, n}^{\top}\right) \boldsymbol{u}(t)=x_{1} h^{*}+o\left(\|\boldsymbol{x}\| \mathrm{e}^{-\gamma t}\right) .
$$

In particular, for $1 \leq i \leq k$, it follows that $q_{i}(t)=c_{i} \mathrm{e}^{-r t}\left(1+o\left(\mathrm{e}^{-\gamma t}\right)\right)$, where

$$
c_{i}=\left(\boldsymbol{e}_{i}^{\top} \boldsymbol{b}_{1}\right) h^{*}=b_{1 i} h^{*}>0,
$$

$\boldsymbol{e}_{i}$ is a column vector with 1 in the $i$ th position and 0 s elsewhere, and $\boldsymbol{b}_{1}$ is the right eigenvector of $\boldsymbol{B}$ corresponding to the eigenvalue $-r$ such that $\boldsymbol{f}_{1}^{\top} \boldsymbol{b}_{1}=1$.

Remark 3.1. The order of convergence is simplified for clarity in the statement of Theorem 3.1. For the case where $\boldsymbol{B}$ is diagonalizable, the exact formulation is as follows. If $-\beta_{2}$ is the second largest real part of an eigenvalue of $\boldsymbol{C}$ and if $r \alpha \neq \beta_{2}$, then $q_{i}(t)=c_{i} \mathrm{e}^{-r t}\left(1+O\left(\mathrm{e}^{-\gamma t}\right)\right)$, where $\gamma=\min \left\{r \alpha, \beta_{2}\right\}$. Otherwise, if $r \alpha=\beta_{2}$ then $q_{i}(t)=c_{i} \mathrm{e}^{-r t}\left(1+O\left(t \mathrm{e}^{-r \alpha t}\right)\right)$.

\section{Time to extinction}

If $\mathrm{E}\left(\left\|J_{i}\right\|^{1+\alpha}\right)<\infty$ for some $0<\alpha \leq 1$ and all $1 \leq i \leq k$, Theorem 3.1 implies that, as $t \rightarrow \infty$,

$$
\mathrm{P}_{\boldsymbol{I}}(T>t)=1-\prod_{i=1}^{k}\left(\mathrm{P}_{i}(T \leq t)\right)^{I_{i}}=1-\prod_{i=1}^{k}\left(1-q_{i}(t)\right)^{I_{i}} \sim 1-\prod_{i=1}^{k}\left(1-c_{i} \mathrm{e}^{-r t}\right)^{I_{i}},
$$

where $c_{i}>0(1 \leq i \leq k)$ and $\boldsymbol{I}=\left(I_{1}, \ldots, I_{k}\right)$ with $I_{i}$ the initial number of type $i$ individuals. Define $C_{\boldsymbol{I}}:=\sum_{j=1}^{k} \bar{I}_{j} c_{j}$. The approximation error in (4.1) is controlled by the following result.

Lemma 4.1. Suppose that $\mathrm{E}\left(\left\|J_{i}\right\|^{1+\alpha}\right)<\infty$ for some $0<\alpha \leq 1$ and all $1 \leq i \leq k$. Then, for any $\gamma$ as in Theorem 3.1, there exist $t_{0}, v_{1}<\infty$, not depending on $\boldsymbol{I}$, such that

$$
\left|\prod_{i=1}^{k}\left(1-q_{i}(t)\right)^{I_{i}}-\prod_{i=1}^{k}\left(1-c_{i} \mathrm{e}^{-r t}\right)^{I_{i}}\right| \leq v_{1} C_{\boldsymbol{I}} \exp \left(-\frac{1}{2} C_{\boldsymbol{I}} \mathrm{e}^{-r t}\right) \mathrm{e}^{-(r+\gamma) t}, \quad t \geq t_{0} .
$$

Proof. Denote the approximation error in (4.1) as $\varepsilon^{(1)}(t)$. Choose

$$
t_{1} \geq \frac{1}{r} \max _{1 \leq i \leq k}\left(\log c_{i}\right)_{+}
$$

such that $q_{i}(t) \geq \frac{1}{2} c_{i} \mathrm{e}^{-r t}$ for all $i$ and $t \geq t_{1}$. Using

$$
\left|\prod_{i=1}^{k} A_{i}-\prod_{i=1}^{k} B_{i}\right| \leq \sum_{l=1}^{k}\left|A_{l}-B_{l}\right|\left(\prod_{i=1}^{l-1}\left|A_{i}\right|\right)\left(\prod_{i=l+1}^{k}\left|B_{i}\right|\right)
$$


where $A_{i}=\left(1-q_{i}(t)\right)^{I_{i}}$ and $B_{i}=\left(1-c_{i} \mathrm{e}^{-r t}\right)^{I_{i}}$, it follows that

$$
\varepsilon^{(1)}(t) \leq \exp \left(-\frac{1}{2} C_{\boldsymbol{I}} \mathrm{e}^{-r t}\right) \sum_{i=1}^{k} I_{i}\left\{\frac{\left|1-q_{i}(t)-\left(1-c_{i} \mathrm{e}^{-r t}\right)\right|}{\min \left\{1-q_{i}(t), 1-c_{i} \mathrm{e}^{-r t}\right\}}\right\}, \quad t \geq t_{1} .
$$

Determine $t_{2}$ such that $\min _{1 \leq i \leq k}\left\{\min \left\{1-q_{i}(t), 1-c_{i} \mathrm{e}^{-r t}\right\}\right\} \geq \frac{1}{2}$ for $t \geq t_{2}$. From Theorem 3.1, we have $\left|q_{i}(t)-c_{i} \mathrm{e}^{-r t}\right| \leq K^{*} c_{i} \mathrm{e}^{-(r+\gamma) t}, 1 \leq i \leq k$, for some $K^{*}<\infty$. Hence, for all $t \geq t_{0}:=\max \left\{t_{1}, t_{2}\right\}$,

$$
\varepsilon^{(1)}(t) \leq 2 C_{\boldsymbol{I}} \exp \left(-\frac{1}{2} C_{\boldsymbol{I}} \mathrm{e}^{-r t}\right) K^{*} \mathrm{e}^{-(r+\gamma) t}
$$

for $\gamma$ as in Theorem 3.1. This completes the proof.

A further approximation to the last term in (4.1) is

$$
1-\prod_{i=1}^{k}\left(1-c_{i} \mathrm{e}^{-r t}\right)^{I_{i}} \sim 1-\exp \left(-C_{\boldsymbol{I}} \mathrm{e}^{-r t}\right) .
$$

The approximation error in (4.2) can be bounded as follows (the proof is omitted).

Lemma 4.2. We have

$$
\left|\prod_{i=1}^{k}\left(1-c_{i} \mathrm{e}^{-r t}\right)^{I_{i}}-\exp \left(-C_{\boldsymbol{I}} \mathrm{e}^{-r t}\right)\right| \leq v_{2} C_{\boldsymbol{I}} \exp \left(-C_{\boldsymbol{I}} \mathrm{e}^{-r t}\right) \mathrm{e}^{-2 r t}, \quad t \geq t_{2},
$$

where $t_{2}$ is as for Lemma 4.1 and $v_{2}=\max _{1 \leq i \leq k} c_{i}<\infty$.

Remark 4.1. Lemmas 4.1 and 4.2 imply that

$$
\begin{gathered}
\varepsilon^{(1)}(t) \leq \frac{\nu_{1}}{C_{\boldsymbol{I}}^{\gamma / r}} \max _{x>0}\left\{\mathrm{e}^{-x / 2} x^{1+\gamma / r}\right\}=\frac{\nu_{3}}{C_{\boldsymbol{I}}^{\gamma / r}}, \quad t \geq t_{0}, \\
\varepsilon^{(2)}(t) \leq \frac{4 \nu_{2} \mathrm{e}^{-2}}{C_{\boldsymbol{I}}}=\frac{\nu_{4}}{C_{\boldsymbol{I}}}, \quad t \geq t_{2},
\end{gathered}
$$

with $t_{0}, t_{2}, v_{1}, v_{2}$, and $\gamma$ as before.

Definition 4.1. Define the random variable $\tilde{T}_{\boldsymbol{I}}$ such that $\mathrm{P}\left(\tilde{T}_{\boldsymbol{I}}>t\right)=1-\exp \left(-C_{\boldsymbol{I}} \mathrm{e}^{-r t}\right)$, where $C_{\boldsymbol{I}}=\sum_{i=1}^{k} I_{i} c_{i}$. The random variable $\tilde{T}_{\boldsymbol{I}}$ satisfies

$$
\tilde{T}_{\boldsymbol{I}}=\frac{\log C_{\boldsymbol{I}}}{r}+\frac{1}{r} V
$$

where $V$ has a Gumbel distribution.

Theorem 4.1. Suppose that $\mathrm{E}\left(\left\|J_{i}\right\|^{1+\alpha}\right)<\infty$ for some $0<\alpha \leq 1$ and all $1 \leq i \leq k$. Then, for $t \geq 0$ and with $\gamma<r \alpha$ as in Theorem 3.1, there exists a constant $\nu^{*}<\infty$ such that

$$
\left|\mathrm{P}_{\boldsymbol{I}}(T>t)-\mathrm{P}\left(\tilde{T}_{\boldsymbol{I}}>t\right)\right| \leq \frac{v^{*}}{C_{\boldsymbol{I}}^{\gamma / r}}
$$


D. HEINZMANN

Proof. Remark 4.1 implies that

$$
\left|\mathrm{P}_{\boldsymbol{I}}(T>t)-\mathrm{P}\left(\tilde{T}_{\boldsymbol{I}}>t\right)\right| \leq \frac{\nu_{3}}{C_{\boldsymbol{I}}^{\gamma / r}}+\frac{\nu_{4}}{C_{\boldsymbol{I}}}, \quad t \geq t_{0} .
$$

For $t \leq t_{0}$, we have

$$
0 \leq \mathrm{P}_{\boldsymbol{I}}(T \leq t) \leq \mathrm{P}_{\boldsymbol{I}}\left(T \leq t_{0}\right) \leq \mathrm{P}\left(\tilde{T}_{\boldsymbol{I}} \leq t_{0}\right)+\frac{\nu_{3}}{C_{\boldsymbol{I}}^{\gamma / r}}+\frac{v_{4}}{C_{\boldsymbol{I}}}
$$

and $0 \leq \mathrm{P}\left(\tilde{T}_{\boldsymbol{I}} \leq t\right) \leq \mathrm{P}\left(\tilde{T}_{\boldsymbol{I}} \leq t_{0}\right)=\exp \left(-C_{\boldsymbol{I}} \mathrm{e}^{-r t_{0}}\right)$, completing the proof.

Theorem 4.1 thus shows that

$$
d_{\mathrm{K}}\left(\mathcal{L}\left(T-\frac{\log C_{\boldsymbol{I}}}{r} \mid \boldsymbol{Z}(0)=\boldsymbol{I}\right), \mathcal{L}\left(\frac{V}{r}\right)\right)=O\left(C_{\boldsymbol{I}}^{-\gamma / r}\right) \quad \text { as }\|I\| \rightarrow \infty,
$$

where $d_{K}$ denotes the Kolmogorov distance between the two distributions indicated by $\mathcal{L}$ and $\gamma$ is as in Theorem 3.1.

We now strengthen the mode of convergence. Let $\tilde{f}_{I}$ be the probability density function of $\tilde{T}_{\boldsymbol{I}}$, and let $f_{\boldsymbol{I}}$ be the probability density function of $T$ under $\mathrm{P}_{\boldsymbol{I}}$.

Lemma 4.3. Suppose that $\mathrm{E}\left(\left\|J_{i}\right\|^{1+\alpha}\right)<\infty$ for some $0<\alpha \leq 1$ and all $1 \leq i \leq k$. For all $t \geq t_{0}$, there exists a constant $K<\infty$ such that

$$
\left|f_{\boldsymbol{I}}(t)-\tilde{f}_{\boldsymbol{I}}(t)\right| \leq K C_{\boldsymbol{I}} \mathrm{e}^{-(r+\gamma) t}\left(1+C_{\boldsymbol{I}} \mathrm{e}^{-r t}\right) \exp \left(-\frac{1}{2} C_{\boldsymbol{I}} \mathrm{e}^{-r t}\right),
$$

where $\gamma$ is as in Theorem 3.1 and $t_{0}$ is as in Lemma 4.1.

Proof. From (4.1) we know that $\mathrm{P}_{\boldsymbol{I}}(T \leq t)=\prod_{i=1}^{k}\left(1-q_{i}(t)\right)^{I_{i}}$, and, thus,

$$
f_{\boldsymbol{I}}(t)=\frac{\mathrm{d}}{\mathrm{d} t} \mathrm{P}_{\boldsymbol{I}}(T \leq t)=\mathrm{P}_{\boldsymbol{I}}(T \leq t) \sum_{i=1}^{k}\left(-\frac{I_{i}}{1-q_{i}(t)} \frac{\mathrm{d} q_{i}(t)}{\mathrm{d} t}\right) .
$$

Furthermore,

$$
\tilde{f}_{\boldsymbol{I}}(t)=\frac{\mathrm{d}}{\mathrm{d} t} \mathrm{P}\left(\tilde{T}_{\boldsymbol{I}} \leq t\right)=\mathrm{P}\left(\tilde{T}_{\boldsymbol{I}} \leq t\right) r C_{\boldsymbol{I}} \mathrm{e}^{-r t}
$$

Lemmas 4.1 and 4.2 imply that, for $t \geq t_{0}$,

$$
\left|\mathrm{P}_{\boldsymbol{I}}(T \leq t)-\mathrm{P}\left(\tilde{T}_{\boldsymbol{I}} \leq t\right)\right| \leq K_{1} C_{\boldsymbol{I}} \exp \left(-\frac{1}{2} C_{\boldsymbol{I}} \mathrm{e}^{-r t}\right) \mathrm{e}^{-(r+\gamma) t} \quad \text { for some } K_{1}<\infty .
$$

Then, also, for $t \geq t_{0}$,

$$
\begin{aligned}
\left|\boldsymbol{I}^{\top} \frac{\mathrm{d} \boldsymbol{q}(t)}{\mathrm{d} t}-\sum_{i=1}^{k} \frac{I_{i}}{1-q_{i}(t)} \frac{\mathrm{d} q_{i}(t)}{\mathrm{d} t}\right| & \leq \sum_{i=1}^{k} I_{i}\left|\frac{\mathrm{d} q_{i}(t)}{\mathrm{d} t}\right|\left|\frac{1}{1-q_{i}(t)}-1\right| \\
& \leq K_{2} C_{\boldsymbol{I}} \mathrm{e}^{-2 r t}
\end{aligned}
$$

with $K_{2}<\infty$, since Lemma 2.1 and Corollary 2.1 imply that $\left|\boldsymbol{I}^{\top} \mathrm{d} \boldsymbol{q}(t) / \mathrm{d} t\right| \leq K_{3} C_{\boldsymbol{I}} \mathrm{e}^{-r t}$ with $K_{3}<\infty$, and, for $t \geq t_{0}, 1-q_{i}(t) \geq \frac{1}{2}$ for $1 \leq i \leq k$, implying that

$$
\left|\frac{1}{1-q_{i}(t)}-1\right| \leq 2 q_{i}(t)=O\left(\mathrm{e}^{-r t}\right) \text {. }
$$


Now, since $\mathrm{d} \boldsymbol{q}(t) / \mathrm{d} t=\boldsymbol{B} \boldsymbol{q}(t)-\boldsymbol{A} \boldsymbol{v}(t)$ as in (2.6), we have

$$
\left|\boldsymbol{I}^{\top} \frac{\mathrm{d} \boldsymbol{q}(t)}{\mathrm{d} t}+r C_{\boldsymbol{I}} \mathrm{e}^{-r t}\right|=\left|\boldsymbol{I}^{\top} \boldsymbol{B} \boldsymbol{q}(t)-\boldsymbol{I}^{\top} \boldsymbol{A} \boldsymbol{v}(t)+r C_{\boldsymbol{I}} \mathrm{e}^{-r t}\right| \leq K_{4} C_{\boldsymbol{I}} \mathrm{e}^{-(r+\gamma) t} .
$$

The final inequality in (4.7) with $K_{4}<\infty$ follows because

(a) Equation (3.3) implies that

$$
\left|\boldsymbol{I}^{\top} \boldsymbol{B} \boldsymbol{q}(t)-\boldsymbol{I}^{\top} \boldsymbol{B} \boldsymbol{b}_{1} h^{*} \mathrm{e}^{-r t}\right| \leq K_{5} C_{\boldsymbol{I}} \mathrm{e}^{-(r+\gamma) t} \quad \text { for } t \geq 0 \text { and } K_{5}<\infty ;
$$

(b) Equation (3.4) and the definition of $\boldsymbol{b}_{1}$ give $\boldsymbol{I}^{\top} \boldsymbol{B} \boldsymbol{b}_{1} h^{*}=-C_{\boldsymbol{I}} \boldsymbol{r}$; and

(c) Corollary 2.1 shows that

$$
\left|\boldsymbol{I}^{\top} \boldsymbol{A} \boldsymbol{v}(t)\right| \leq K_{6} C_{\boldsymbol{I}} \mathrm{e}^{-r(1+\alpha) t} \quad \text { for } t \geq 0 \text { and } K_{6}<\infty .
$$

Combining (4.6) and (4.7) thus gives

$$
\left|r C_{I} \mathrm{e}^{-r t}+\sum_{i=1}^{k} \frac{I_{i}}{1-q_{i}(t)} \frac{\mathrm{d} q_{i}(t)}{\mathrm{d} t}\right| \leq K_{7} \mathrm{e}^{-(r+\gamma) t} .
$$

Using (4.5) and (4.8), together with the triangle inequality now applied to the difference of (4.3) and (4.4) in the form

$$
\left|A_{1} A_{2}-B_{1} B_{2}\right| \leq\left|A_{1}-B_{1}\right|\left|A_{2}-B_{2}\right|+\left|B_{2}\right|\left|A_{1}-B_{1}\right|+\left|B_{1}\right|\left|A_{2}-B_{2}\right|,
$$

we obtain the lemma.

Using Lemma 4.3, we can show that the distribution of $T$ under $\mathrm{P}_{I}$ can be well approximated by that of $\tilde{T}_{\boldsymbol{I}}$ in terms of probability densities and the total variation distance $d_{\mathrm{TV}}$.

Theorem 4.2. Suppose that $\mathrm{E}\left(\left\|J_{i}\right\|^{1+\alpha}\right)<\infty$ for some $0<\alpha \leq 1$ and all $1 \leq i \leq k$. Then there exist constants $K_{a}, K_{b}<\infty$ such that

(i) $\left|f_{\boldsymbol{I}}(t)-\tilde{f}_{\boldsymbol{I}}(t)\right| \leq K_{a} C_{\boldsymbol{I}}^{-\gamma / r}, t \geq 0$;

(ii) $d_{\mathrm{TV}}\left(\mathcal{L}(T \mid \boldsymbol{Z}(0)=\boldsymbol{I}), \mathcal{L}\left(\tilde{T}_{\boldsymbol{I}}\right)\right)=\frac{1}{2} \int_{0}^{\infty}\left|f_{\boldsymbol{I}}(t)-\tilde{f}_{\boldsymbol{I}}(t)\right| \mathrm{d} t \leq K_{b} C_{\boldsymbol{I}}^{-\gamma / r}$.

Proof. For $t \geq t_{0}$, part (i) follows from Lemma 4.3, since $x^{1+\gamma / r}(1+x) \mathrm{e}^{-x / 2}$ is uniformly bounded in $x \geq 0$. For $t \leq t_{0}$, we have

$$
\tilde{f}_{\boldsymbol{I}}(t) \leq C_{\boldsymbol{I}} r \exp \left(-C_{\boldsymbol{I}} \exp \left(-r t_{0}\right)\right)=O\left(C_{\boldsymbol{I}}^{-s}\right) \text { for all } s>0 ;
$$

similarly, from (4.1) and (4.3), it can be shown that

$$
f_{\boldsymbol{I}}(t) \leq K C_{\boldsymbol{I}} \exp \left(-\sum_{i=1}^{k} d_{i} I_{i}\right)
$$

with $d_{i}=-\log \left(1-q_{i}\left(t_{0}\right)\right)>0(1 \leq i \leq k)$ and $K<\infty$, which is also of order $O\left(C_{\boldsymbol{I}}^{-s}\right)$ for all $s$, completing the proof of part (i).

For part (ii), by Lemma 4.3,

$$
\int_{t_{0}}^{\infty}\left|f_{\boldsymbol{I}}(t)-\tilde{f}_{\boldsymbol{I}}(t)\right| \mathrm{d} t \leq K_{c} C_{\boldsymbol{I}}^{-\gamma / r}
$$

for $K_{c}<\infty$ a constant. The remaining part is bounded using part (i). 


\section{Application}

Theorem 4.1 is illustrated by a two-type model for parasitic resistance, in which the parasite can enter a resting phase during which it does not reproduce, but can be transmitted easily to a new host. An example is the transmission cycle of the parasitic protozoa Toxoplasma gondii [6] in the intermediate hosts, which are warm-blooded. One third of the world's human population is estimated to carry a Toxoplasma infection [15]. The growth rate of a parasite population within the intermediate host can be modeled by a two-type continuous-time Markov branching process. A parasite is of type 1 if it is in the active state and of type 2 if it is in the resting state. A type 1 parasite can either die at rate $d_{1}$, enter the resting state at rate $r_{1}$, or reproduce itself by binary splitting at rate $\rho$. A type 2 parasite can either die at rate $d_{2}$ or become active within the host by changing to the active state at rate $r_{2}$. The transmission of the parasite to another host is incorporated in the death event. All interevent times are exponentially distributed.

Let $Z_{i}=Z_{i}(t)(i=1,2)$ be the number of type $i$ parasites in a host at time $t \geq 0$. The transition scheme of the process is given in Table 1 .

Let $a_{1}:=d_{1}+r_{1}+\rho$ and $a_{2}:=d_{2}+r_{2}$ be the total rates of transition for type 1 and type 2 individuals, respectively. For $q_{i}(t)(i=1,2)$, system (2.2) yields

$$
\frac{\mathrm{d} \boldsymbol{q}(t)}{\mathrm{d} t}=\left(\begin{array}{cc}
\left(-a_{1}+2 \rho\right) & r_{1} \\
r_{2} & -a_{2}
\end{array}\right) \boldsymbol{q}(t)-\left(\begin{array}{c}
\rho q_{1}(t)^{2} \\
0
\end{array}\right)=\boldsymbol{B} \boldsymbol{q}(t)-\boldsymbol{v}(t) .
$$

Since $\|\boldsymbol{J}\| \leq 2$ for $\boldsymbol{Z}(0)=(1,0)^{\top}$ and $\boldsymbol{Z}(0)=(0,1)^{\top}$, we can take $\alpha=1$.

Theorem 2.1 implies that $\boldsymbol{B}$ has a unique real largest eigenvalue $-r$, with corresponding positive left, $\boldsymbol{f}_{1}^{\top}$, and right, $\boldsymbol{b}_{1}$, eigenvectors, which are given by

$$
\begin{gathered}
-r=\frac{-\left(a_{1}-2 \rho+a_{2}\right)+\sqrt{D}}{2}, \\
f_{1}^{\top}=\frac{1}{N_{1}}\left(\frac{a_{2}+2 \rho-a_{1}+\sqrt{D}}{2 r_{1}}, 1\right), \\
\text { and } \boldsymbol{b}_{1}^{\top}=\frac{1}{N_{2}}\left(\frac{a_{2}+2 \rho-a_{1}+\sqrt{D}}{2 r_{2}}, 1\right),
\end{gathered}
$$

where

$$
D=\left(\left(a_{1}-2 \rho+a_{2}\right)^{2}-4\left(a_{2}\left(a_{1}-2 \rho\right)-r_{1} r_{2}\right)\right),
$$

$N_{1}$ and $N_{2}$ are appropriate constants such that $\left|\boldsymbol{f}_{1}\right|=1$ and $\boldsymbol{f}_{1}^{\top} \boldsymbol{b}_{1}=1$.

The process $(\boldsymbol{Z}(t))_{t \geq 0}$ is subcritical if and only if (i) $a_{2}\left(a_{1}-2 \rho\right)>r_{1} r_{2}$ and (ii) $a_{1}-2 \rho$ $+a_{2}>0$. Let the model parameters be fixed as in Table 2 such that the process is subcritical.

TABLE 1.

\begin{tabular}{cc}
\hline Transition & Rate \\
\hline$Z_{1} \rightarrow Z_{1}-1, Z_{2} \rightarrow Z_{2}$ & $d_{1} Z_{1}$ \\
$Z_{1} \rightarrow Z_{1}-1, Z_{2} \rightarrow Z_{2}+1$ & $r_{1} Z_{1}$ \\
$Z_{1} \rightarrow Z_{1}+1, Z_{2} \rightarrow Z_{2}$ & $\rho Z_{1}$ \\
$Z_{1} \rightarrow Z_{1}, Z_{2} \rightarrow Z_{2}-1$ & $d_{2} Z_{2}$ \\
$Z_{1} \rightarrow Z_{1}+1, Z_{2} \rightarrow Z_{2}-1$ & $r_{2} Z_{2}$ \\
\hline
\end{tabular}


TABLE 2: Accuracy of the extinction time approximation of the two-type Markov branching process (Table 1). For given $\boldsymbol{I}=\left(I_{1}, I_{2}\right)$, the approximation $\tilde{T}_{\boldsymbol{I}}$ is compared to $T$ (500000 simulations) by computing the proportion of simulated values of $T$ larger than or equal to the median $\tilde{m}$ of $\tilde{T}_{\boldsymbol{I}}$, and by calculating the proportion of simulated values of $T$ falling into the interquartile range (IQR) defined as the interval $\left(\tilde{q}_{1}, \tilde{q}_{3}\right)$, where $\tilde{q}_{1}$ and $\tilde{q}_{3}$ are the first and third quartiles of the approximating distribution of $\tilde{T}_{I}$. The results are displayed for $I_{1}: I_{2}=5: 1$ and $2: 1$ and different values of $I_{1}$. The corresponding $C_{\boldsymbol{I}}=c_{1} I_{1}+c_{2} I_{2}$ are also represented. The model parameters are set to $\left(d_{1}, r_{1}, \rho\right)=(1,1,0.5)$ and $\left(d_{2}, r_{2}\right)=(1.2,0.8)$ such that $-r<0$.

\begin{tabular}{lcccccc}
\hline$I_{1}=5 I_{2}$ & 10 & 50 & 100 & 500 & 1000 & 5000 \\
\hline$C_{\boldsymbol{I}}$ & 9.618 & 48.090 & 96.179 & 480.897 & 961.793 & 4808.966 \\
\hline $\mathrm{P}(\geq \tilde{m})$ & 0.516 & 0.503 & 0.501 & 0.501 & 0.498 & 0.500 \\
$\mathrm{IQR}$ & 0.529 & 0.506 & 0.504 & 0.501 & 0.500 & 0.500 \\
\hline$I_{1}=2 I_{2}$ & 10 & 50 & 100 & 500 & 1000 & 5000 \\
\hline$C_{\boldsymbol{I}}$ & 11.342 & 56.711 & 113.422 & 567.111 & 1134.221 & 5671.105 \\
\hline $\mathrm{P}(\geq \tilde{m})$ & 0.514 & 0.503 & 0.502 & 0.499 & 0.500 & 0.500 \\
$\mathrm{IQR}$ & 0.525 & 0.504 & 0.503 & 0.501 & 0.499 & 0.500 \\
\hline
\end{tabular}

Thus, $r=0.821, \beta_{2}=1.857$, and $\alpha=1$, and Remark 3.1 implies that

$$
q_{i}(t)=c_{i} \mathrm{e}^{-r t}\left(1+O\left(\mathrm{e}^{-r t}\right)\right) .
$$

Furthermore, the Kolmogorov and the total variation distances between the distributions of $T$ given $\boldsymbol{Z}(0)=\boldsymbol{I}$ and of $\tilde{T}_{\boldsymbol{I}}$ are both of order $O\left(C_{\boldsymbol{I}}^{-1}\right)$. To compute $c_{i}=\left(\boldsymbol{e}_{i}^{\top} \boldsymbol{b}_{1}\right) h^{*}(i=1,2)$, it is necessary to determine $h^{*}$, given by

$$
\log h^{*}:=\lim _{t \rightarrow \infty}\left\{\log \left(\boldsymbol{f}_{1}^{\top} \boldsymbol{q}(t)\right)+r t\right\} .
$$

This entails the numerical solution of system (5.1) up to a sufficient large $t$. To increase the numerical stability, it is advisable to solve for $\mathrm{e}^{r t} \boldsymbol{q}(t)$ instead of $\boldsymbol{q}(t)$ by appropriately reformulating (5.1). To determine an appropriate $t$, the reformulated system is successively solved for $t \in\{10,11,12, \ldots\}$, and the corresponding $c_{1}$ and $c_{2}$ are evaluated until the absolute differences of successive values of $c_{1}$ and $c_{2}$ are both smaller than some predefined level, $10^{-10}$ in our example, resulting in $c_{1}=0.847$ and $c_{2}=0.575$ at $t=16$.

Given $\boldsymbol{Z}(0)=\boldsymbol{I}=\left(I_{1}, I_{2}\right)$, the distribution of $\tilde{T}_{\boldsymbol{I}}$ given in Definition 4.1 can be compared with the distribution of the true extinction time $T$, which has to be computed by simulation, since the exact result is inaccessible. For the simulation, the Markov chain (see Table 1) can be simulated by the classical Gillespie algorithm [8] or an improved version thereof [9].

Table 2 indicates a location and a scale measure for evaluating the approximation performance. The closeness of the probabilities $\mathrm{P}_{\boldsymbol{I}}(T>\tilde{m})$ and $\mathrm{P}_{\boldsymbol{I}}\left(\tilde{q}_{1}<T<\tilde{q}_{3}\right)$ to their limiting values 0.5 , where $\tilde{m}, \tilde{q}_{1}$, and $\tilde{q}_{3}$ are the median, the first, and the third quartiles of the approximating distribution of $\tilde{T}_{I}$, increases with higher values of $C_{I}$, which is in line with the previous results. Figure 1 represents the density function of the approximated extinction time versus the true extinction time for different initial configurations $\boldsymbol{I}=\left(I_{1}, I_{2}\right)$. The density of the approximated distribution closely matches the distribution of the simulated times, supporting the results in this paper. 

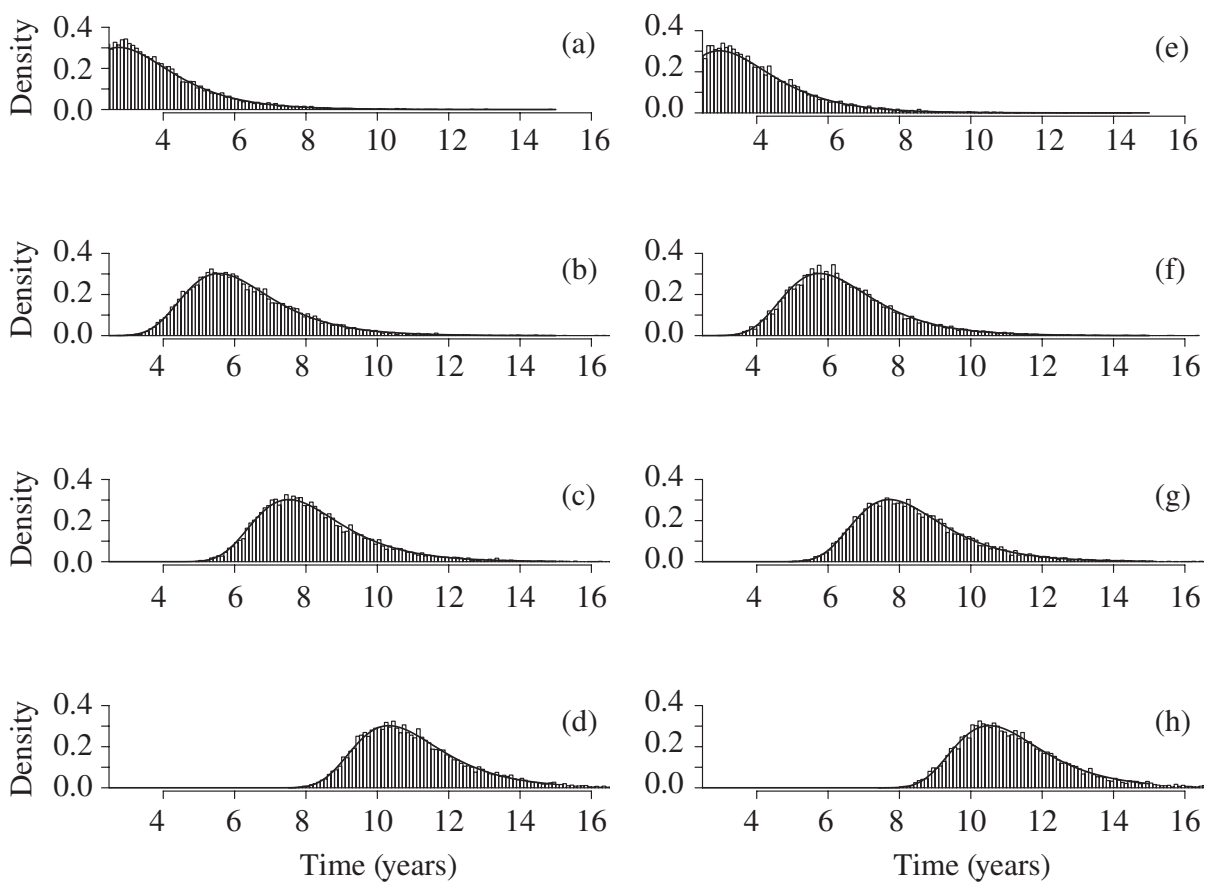

Figure 1: The density distribution of $\tilde{T}_{\boldsymbol{I}}$ (solid line) versus the simulated distribution of $T$ (histogram of 10000 simulations) for ratios $I_{1}: I_{2}=5: 1((\mathrm{a})-(\mathrm{d}))$ and $2: 1$ ((e)-(h)) with $I_{1}$ equal to 10 ((a) and (e)), $100((\mathrm{~b})$ and (f)), $500((\mathrm{c})$ and $(\mathrm{g}))$, and $5000((\mathrm{~d})$ and $(\mathrm{h}))$. The model parameters are fixed as in Table 2.

\section{Acknowledgements}

The author wishes to thank Andrew Barbour for many helpful suggestions and comments. The author also gratefully acknowledges the anonymous referee for suggestions that greatly improved the presentation. This work was supported by the Schweizerischer Nationalfonds (SNF), project number 107726.

\section{References}

[1] Athreya, K. B. And Ney, P. E. (1972). Branching Processes. Springer, New York.

[2] Ball, F. (1983). The threshold behaviour of epidemic models. J. Appl. Prob. 20, 227-241.

[3] Ball, F. and Donnelly, P. (1995). Strong approximations of epidemic models. Stoch. Process. Appl. 55, 1-21.

[4] Barbour, A. D. (2007). Coupling a branching process to an infinite dimensional epidemic process. Preprint. Available at http://arxiv.org/abs/0710.3697v1.

[5] Barbour, A. D. and Utev, S. (2004). Approximating the Reed-Frost epidemic process. Stoch. Process. Appl. 113, 173-197.

[6] Eckert, J., Friedhoff, K. T., Zahner, H. And Deplazer, P. (eds) (2005). Lehrbuch der Parasitologie für die Tiermedizin. Enke Verlag, Stuttgart.

[7] Galambos, J. and Simonelli, I. (1996). Bonferroni-type Inequalities with Applications. Springer, New York.

[8] Gillespie, D. T. (1977). Exact stochastic simulation of coupled chemical reactions. J. Chem. Phys. 81, 23402361.

[9] Gillespie, D. T. and Petzold, L. R. (2003). Improved leap-size selection for accelerated stochastic simulation. J. Chem. Phys. 119, 8229-8234.

[10] Gronwall, T. H. (1919). Note on the derivatives with respect to a parameter of the solutions of a system of differential equations. Ann. Math. 20, 292-296.

[11] Harris, T. E. (1963). The Theory of Branching Processes. Springer, Berlin.

[12] Jagers, P. (1975). Branching Processes with Biological Applications. John Wiley, New York. 
[13] Jagers, P., Klebaner, F. C. And Sagitov, S. (2007). On the path to extinction. Proc. Nat. Acad. Sci. USA 104, 6107-6111.

[14] LeVinson, N. (1948). The asymptotic nature of solutions of linear systems of differential equations. Duke Math. J. 15, 111-126.

[15] Montoya, J. G. And Liesenfeld, O. (2004). Toxoplasmosis. The Lancet 363, 1965-1976.

[16] Seneta, E. (1973). Non-negative Matrices. Halsted Press, New York.

[17] Sewastuanow, B. A. (1974). Verzweigungsprozesse. Akademie, Berlin.

[18] Whittle, P. (1955). The outcome of a stochastic epidemic_a note on Bailey's paper. Biometrika 42, 116-122. 